\title{
Quelle attitude adopter face aux cadeaux et invitations à dîner
}

\author{
Bruno Henggi ${ }^{a}$, Charlotte Schweizer ${ }^{b}$ \\ ${ }^{a}$ Responsable Affaires publiques de la $\mathrm{FMH} ;{ }^{b}$ Cheffe de la division Communication
}

Les nouvelles prescriptions sur l'utilisation des produits thérapeutiques qui entrent en vigueur le $1^{\text {er }}$ janvier 2020 comportent des règles plus strictes et ont un impact direct sur le travail quotidien des médecins. Elles sont définies dans l'ordonnance sur l'intégrité et la transparence dans le domaine des produits thérapeutiques (OITPTh), qui vise à renforcer l'intégrité et à augmenter la transparence dans la manière de gérer les produits thérapeutiques. Elle règle donc en détail les rabais et compensations de la part des fournisseurs de médicaments. De plus, elle comprend des dispositions relatives aux dons destinés à la recherche et à la formation postgraduée et continue. Elle prévoit également des règles strictes pour ce qui concerne l'acceptation de cadeaux et invitations à des repas.

Pour les médecins, cela signifie se familiariser avec les dispositions de la nouvelle ordonnance afin de pouvoir les respecter et les appliquer correctement, mais aussi documenter leurs actes de manière systématique et complète. En tant qu'association professionnelle, la FMH considère qu'il est de son devoir de rendre ses membres attentifs à cette réglementation plus stricte et de les informer plus largement des nouvelles dispositions légales. Nous comptons le faire dans le cadre d'une série en quatre volets.

Dans le présent article, nous nous intéressons de plus près aux questions se posant dans le contexte des cadeaux et invitations à dîner. Nous les traitons à l'aide d'exemples pratiques tels qu'ils peuvent survenir dans le quotidien des médecins.

\section{Invitations à dîner}

Question: Un professionnel reçoit une invitation à dîner de la part d'une entreprise pharmaceutique. Dans quelles circonstances peut-il accepter une telle invitation et, plus exactement, dans quelles conditions la prise en charge des frais de repas par l'entreprise estelle légale?

Réponse: Le dîner mentionné doit inclure un entretien professionnel. Cela signifie que l'entretien porte sur un thème pertinent pour l'entreprise pharmaceutique et qu'il génère donc de la valeur ajoutée. Juridiquement, il n'existe pas encore de définition plus précise de l'«entretien professionnel». Il est cependant certain que cet entretien est lié à une contrepartie professionnelle. Dans ce cas, les médecins peuvent accepter une invitation jusqu'à un maximum de 100 francs. $\mathrm{Au}$-delà de cette valeur, une invitation n'est pas admise, à moins qu'elle ait fait l'objet d'une convention écrite stipulant prestation et contrepartie. Pour respecter la limite des 100 francs, il est recommandé de tenir compte des prix sur le menu et la carte des vins. Par précaution, le professionnel peut documenter le dîner dans une note d'entretien.

\section{Base légale de l'OITPTh: explication}

Les médecins peuvent donc accepter des invitations à dîner sans convention écrite et se faire payer leur repas, à condition que cela se déroule dans le cadre d'un entretien professionnel et que les frais de repas, boissons incluses, ne dépassent pas 100 francs. Les invitations à dîner ne sont pas admises si elles se déroulent sans entretien professionnel (à titre de contrepartie) ou si la valeur du repas, boissons incluses, est supérieure à 100 francs et qu'aucune convention écrite ne vient préciser la prestation accordée en contrepartie. 


\section{Texte de l'ordonnance}

Art. 7 Compensations accordées en contrepartie de prestations équivalentes

1 Les compensations au sens de l'art. 55, al. 2, let. c, LPTh accordées à des professionnels ou à des organisations en contrepartie de prestations équivalentes sont admises pour autant qu'elles:

a. se fondent sur une convention écrite indiquant la nature et l'ampleur de la prestation et de la compensation, et qu'elles

b. soient proportionnées à la prestation.

2 Dans le cadre d'un entretien professionnel, la prise en charge de frais de repas jusqu'à un maximum de 100 francs est exemptée de l'obligation visée à l'al. 1, let. $a$.

\section{Cadeaux et rémunérations}

Question: Dans quelles circonstances un professionnel peut-il accepter un cadeau d'une entreprise pharmaceutique?

Réponse: Les cadeaux sans rapport avec la pratique de la médecine ou de la pharmacie sont explicitement interdits, et notamment les bouteilles de vin ou de spiritueux, mais aussi des invitations ou billets d'entrée à des manifestations culturelles. Les cadeaux de valeur modeste qui ont un rapport avec la pratique de la médecine ou de la pharmacie sont en revanche admis. Cela signifie, d'une part, que la valeur du cadeau par professionnel ou cabinet médical (pour plusieurs professionnels) ne doit pas dépasser 300 francs par an. Pour les cabinets individuels, le montant est de 300 francs par médecin et par an. Pour les cabinets de groupe, les 300 francs constituent la valeur limite globale cumulée de tous les médecins du même cabinet. D'autre part, le cadeau doit être en rapport avec l'activité du professionnel ou bénéficier directement aux patients. Sont par exemple admis:

- Littérature spécialisée ou formation postgraduée et continue au moyen de médias électroniques tels que supports d'images, de son ou de données.

- Outils de travail tels que thermomètres, logiciels ou téléphones portables pour le service d'urgences (le prix des portables ne doit pas dépasser 300 francs).

- Equipements pour le cabinet, par exemple distributeur d'eau minérale pour les patients ou articles de salle d'attente tels que magazines ou jouets pour enfants. Il faut cependant tenir compte du fait que les fournisseurs de médicaments peuvent essayer de placer des cadeaux en tant que support publicitaire et proposer aux médecins une indemnisation soi-disant légale sous forme de contrat. Nous mettons en garde contre de telles offres, car le cadeau ne correspond plus dans ce cas aux conditions d'exception décrites. Nous recommandons de faire examiner ces offres par un juriste.

Les cadeaux mentionnés et admis lorsqu'ils ont un rapport avec la pratique de la médecine ou de la pharmacie n'ont pas besoin de faire l'objet d'une note écrite.

\section{Exemples concrets de cadeaux}

Livre d'images pour enfants: Un médecin reçoit un nouveau livre d'images pour enfants sur le cholestérol à déposer dans sa salle d'attente.

Question: Le médecin peut-il accepter ce cadeau? Réponse: Oui, car cet avantage bénéficie aux patients. Le médecin doit cependant déposer le livre dans la salle d'attente.

Cheval à bascule: Un médecin reçoit un cheval à bascule à mettre dans sa salle d'attente pour que les enfants de ses patients puissent jouer. Sur le cheval figure l'inscription "Made in Switzerland» et une étiquette indiquant «Produit fabriqué à la main, de la plus haute qualité suisse».

Question: Le médecin peut-il accepter ce cadeau? Réponse: Oui, car cet avantage bénéficie aux patients. Le médecin doit cependant le mettre à disposition dans la salle d'attente.

Bon cadeau: Lors du congrès d'une entreprise pharmaceutique, un médecin gagne un bon cadeau pour une nuit à l'hôtel Mont-Cervin à Zermatt.

Question: Le médecin peut-il accepter le bon cadeau? Réponse: Non, cet avantage gagné dans le cadre d'un concours n'est pas admis, car sa valeur n'est pas modeste et il n'a pas de rapport avec la pratique de la médecine ou de la pharmacie. Un gain admis dans le cadre d'un concours serait par exemple de la littérature spécialisée d'une valeur inférieure à 300 francs par cabinet médical et par an.

\section{Visites de représentants pharmaceutiques}

Le représentant d'une entreprise pharmaceutique présente les nouveaux médicaments dans le cadre d'une visite au cabinet. Il apporte des pâtisseries pour tous les employés du cabinet.

Question: Ce genre d'attention peut-il être accepté? Réponse: Les dispositions relatives aux avantages s'appliquent aussi aux personnes qui ne prescrivent pas de médicaments sous leur propre responsabilité 
et aux employés du cabinet (assistantes médicales). Ce genre de petits présents plein de bonnes intentions sont donc problématiques. Les pâtisseries apportées par un représentant doivent être offertes aux patients dans la salle d'attente ou refusées.

\section{Base légale de l'OITPTh: explication}

Le but de la nouvelle ordonnance est d'éviter que la prescription, la remise, l'utilisation ou l'achat de médicaments soumis à ordonnance soient influencés de quelque manière que ce soit. Au lieu d'imposer une interdiction totale de toute marque d'attention, la nouvelle ordonnance établit une liste des exceptions qui indique de manière exhaustive ce qui est juridiquement encore possible.

Cela inclut les avantages de valeur modeste qui sont limités à 300 francs par an et par professionnel ou cabinet médical. Pour les cabinets de groupe, la limite de 300 francs s'applique à tous les médecins travaillant dans le cabinet. Il est important de noter que ces avantages doivent avoir un rapport avec la pratique de la médecine. Il peut s'agir de littérature spécialisée, d'instruments tels qu'un thermomètre, de logiciels ou d'un téléphone portable pour le service d'urgences. Cela inclut aussi les distributeurs d'eau minérale pour les patients, des jouets pour enfants ou des magazines pour salle d'attente. Les cadeaux personnels en tout genre ne sont pas admis s'ils n'ont aucun lien avec l'exercice de la profession et ne bénéficient donc pas aux patients.

Les gains et lots de concours sont admis s'ils ont une valeur modeste et s'ils sont en rapport avec la pratique de la médecine ou de la pharmacie. Par ailleurs, le concours ne peut s'adresser qu'aux professionnels visés par la publicité qui leur est destinée et la participation au concours ne peut pas être liée à l'achat de médicaments soumis à ordonnance.

\section{Texte de l'ordonnance}

\section{Section 2 Intégrité}

\section{Art. 3 Avantages de valeur modeste}

1 Les avantages aux professionnels dont le montant total ne dépasse pas 300 francs par professionnel et par an et qui ont un rapport avec la pratique de la médecine ou de la pharmacie sont admis comme avantages de valeur modeste au sens de l'art. 55, al. 2, let. a, LPTh.

2 Un avantage est réputé avoir un rapport avec la pratique de la médecine ou de la pharmacie lorsqu'il est directement lié à l'activité du professionnel, ou lorsqu'il bénéficie directement aux clients ou aux patients du professionnel.

3 Les gains et les lots de concours ne sont admis qu'à condition:

a. qu'ils consistent en un avantage de valeur modeste et en rapport avec la pratique de la médecine ou de la pharmacie;

$b$. que le concours s'adresse exclusivement aux personnes visées par la publicité destinée aux professionnels au sens de l'art. 3 de l'ordonnance du 17 octobre 2001 sur la publicité pour les médicaments (OPUM), et

c. que la participation ne soit pas liée à l'achat de médicaments soumis à ordonnance.

\section{Recommandation}

En conclusion, la FMH recommande aux médecins de prendre à l'avenir certaines précautions lors de livraisons de médicaments. Les médecins doivent tenir compte du fait que les cadeaux et petits présents sans rapport avec la pratique de la médecine ou de la pharmacie sont explicitement interdits et doivent donc être refusés. Par ailleurs, leur valeur ne doit pas dépasser 300 francs par an et par professionnel ou cabinet.

Il est également important de noter que les invitations à dîner ne doivent pas dépasser une valeur de 100 francs, boissons incluses, et qu'il est dans l'intérêt du médecin de contrôler que cette limite soit respectée. 